\title{
LICÓFITAS PALEOZÓICAS DO CHILE
}

\author{
M.E. BERNARDES DE OLIVEIRA \\ O. RÖSLER \\ Departamento de Paleontologia e \\ Estratigrafia, IGUSP
}

\section{RESUMO}

Impressões caulinares de licófitas da Formação Arrayán, do Neopaleozóico da Cordilheira Chilena são aqui estudadas. Seu carater fragmentário e sua má preservação nāo permitem uma identificação genérica segura. Entretanto foram reconhecidos 5 tipos básicos de cicatrizes os quais apresentam relaçoes possiveis com Haplostigma Seward, Cyclostigma pacifica Jongmans, Bergeria Presl e mais distantemente Protolepidodendron e Sigillaria do tipo Eusigillaria. São feitas algumas reflexões em relação a uma possivel idade $\mathrm{D}$ superior a $\mathrm{C}$ inferior.

\section{INTRUDUÇÃO}

$\mathrm{Na}$ região andina chilena, segundo AGUIRRE et alii (1974:2), o dobramento do embasamento no Mioceno Superior e o falhamento normal no Plio-Pleistoceno, produziram 5 unidades morfoestruturais de direção nortesul, a saber de W para E: Cordilheira Costeira, Vale Central, Cordilheira Principal, Cordilheira Frontal e Precordilheira. Vide Fig. 1.

Essencialmente o que se tem é o seguinte: A Serie Andina (meso-cenozóica), que aflora ao longo da vertente oriental da Cordilheira Costeira e ao longo da Cordilheira Principal, constitui um amplo sinclinório suspenso de eixo N-S, inserido entre dois anticlinórios paleozóicos do embasamento, ou seja, a vertente ocidental da Cordilheira Costeira (paleozóica) e a Cordilheira Frontal (neopaleozóica). Esta última unidade repousa ao $\mathrm{N}$ do paralelo de 33 \% $\mathrm{S}$, sobre a Precordilheira, que constituiria um 3 ọ eixo, Eopaleozóico. O Vale Central, interpretado como um graben, aparece entre a Cordilheira Costeira e a Principal, ao S do paralelo de 33 o S, portanto, é constituido de terrenos meso-cenozóicos.

Os fósseis aqui estudados são provenientes da Formaçāo Arrayán, constituinte da unidade morioestrutural do flanco ocidental da Cordilheira Costeira, portanto, do embasamento paleozoivo dos Andes.

A Formacão Arrayan é constituida de are- nitos cuja matriz é de mesma composição que seus grãos, sendo interpretada por Reutter, Charrier e outros autores, (apud CECIONI 1974) como depósitos de flysch. Tais depósitos segundo CECIONI ter-se-iam precipitado em ambiente lacustre, baseado no fato de conterem restos de plantas terrestres, apresentarem matriz de mesma composição que os grãos e ausência de fósseis marinhos.

CECIONI (1974) acredita que esses depósitos teriam como àrea fonte depósitos do Devoniano Inferior, que uma vez diagenizados teriam sido soerguidos, formando escarpas, cujos escombros, após pequeno arraste, se depositariam em lagos do Devoniano Superior.

Esse flysch seria sin-orogenético e se correlacionaria com o flysch devoniano da Precorditheira dos Andes Centrais argentino-chilenos. Teria se formado por repetidas correntes de turbidez para dentro do ambiente lacustre, produzidas por diastrofismo.

Ainda segundo CECIONI (1974) a Formação Arrayán acha-se recoberta pelo Membro Desembocadura da Formação Huentelauquén (Pensilvaniano Médio) tendo sido afetada por uma orogenese anterior a esse Membro, identificada como de subfase Bretonica da fase Acadiana (a última do diastrofismo caledoniano).

O material estudado foi coletado em 1976 por uma equipe constituida pelos Dr. R. Charrier, Dr. F. Hervé A. e pelos autores do pre- 
sente trabalho. Está depositado na Coleção Pa. leomtológica do Instituto de Geociências da USP e consiste de 13 amostras registradas sob os nos GP/3T-1201 a GP/3T-1213. Procedem de afloramento da Formação Arrayán. Esse afloramento apresenta-se como um grande paredão de arenito de estratificação gradacional, bastante micáceo, de coloração verde-cinzento.

\section{CONTEÚDO FOSSILIIFERO}

Tratam-se de caules e umas raras folhas dispersas de licófitas fossilizadas sob a forma de impressōes e compressões extremamente fragmentadas.

Esse aspecto fragmentário apresentado separadamente diferentes alturas de um mesmo caule, associado a um estado de decortição muito variável, a pobreza de detalhes mais finos numa matriz relativamente grosseira têm tornado quase impossível uma identificação segura mesmo a nivel genérico.
Dos espécimes observados, com exceção do GP/3T-1213, que apresenta folhas dispersas, todos os demais podem ser agrupados em 5 tipos básicos de cicatrizes foliares, que contudo em alguns casos são até coexistentes no mesmo individuo.

\section{Cicatrizes Foliares Tipo A}

Est. I, Fig 1 e 2

\section{Espécimes: GP/3T-1201 e GP/3T. 1202}

Descriçāo: Cicatrizes foliares de aspecto arredondado a oval transversalmente, de 1 a $1,5 \mathrm{~mm}$ de diâmetro, com feixe vascular de posição central razoavelmente nítido. As estriaçōes finas entre as cicatrizes são transversais em relação ao alongamento do caule. As cicatrizes estâo dispostas numa helicoidal de ângulo muito pequeno em relação à horizontal, dando um aspecto pseudoverticilado. A distância entre uma cicatriz foliar

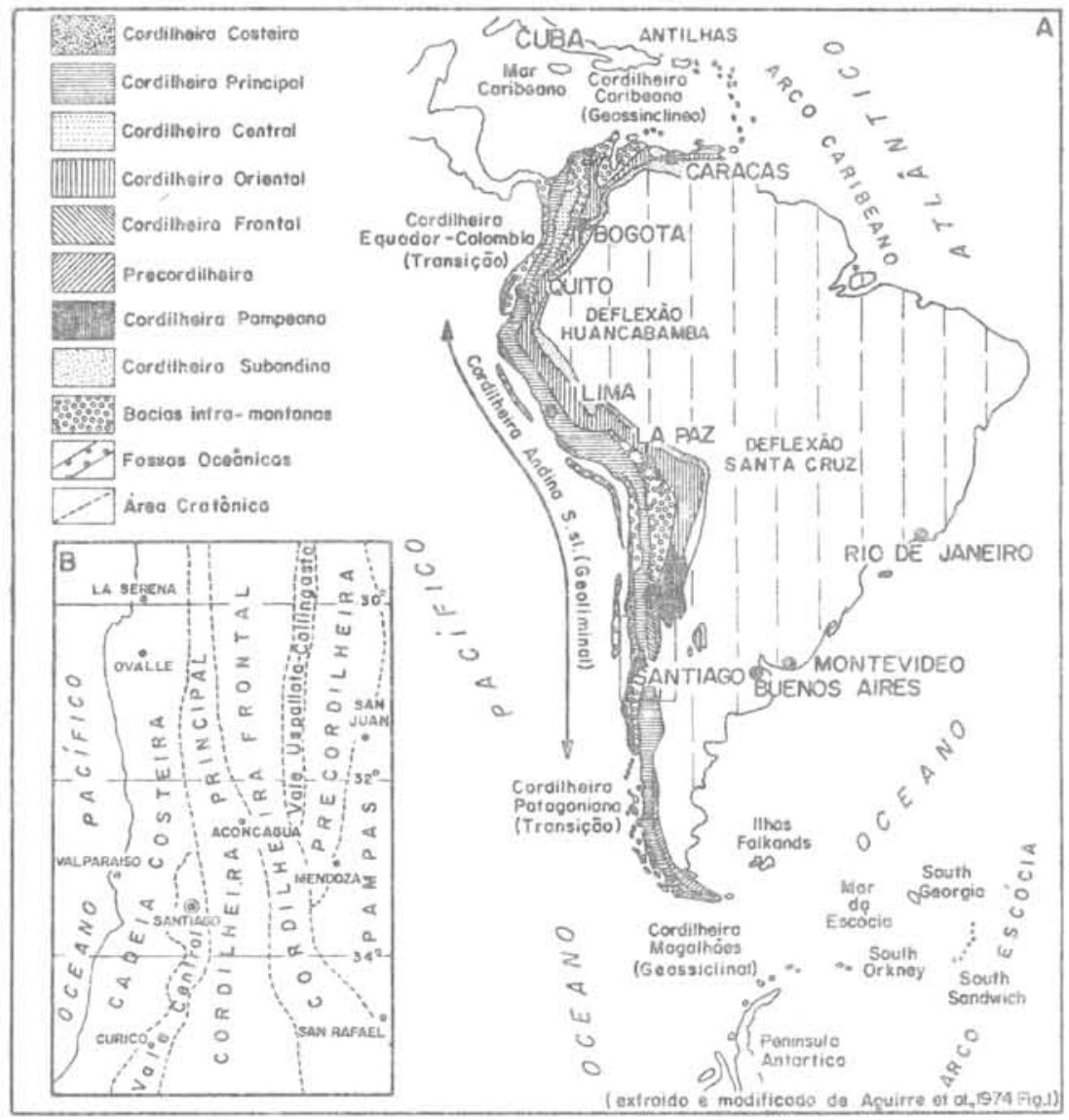

Fig. I UNIDADES MORFO - ESTRUTURAIS DO SISTEMA OROGÊNICO DO PACIFICO SUDESTE. 
e a próxima, na sequência helicoidal, é de cerca de 2,5 a $2,8 \mathrm{~mm}$. Não se observam almofadas foliares, paricnos e lígula. Na sequência longitudinal a distância entre as cicatrizes é de $4 \mathrm{~mm}$.

Comparação: Pela forma de suas cicatrizes, pelo distanciamento entre elas e pelas estriação transversal assemelha-se a Haplostigma Seward de Plumstead (1967: PL XIV, fig. 4 e 5).

\section{Cicatrizes Foliares Tipo B}

Est. I fig 3 a 8 ,

Espécimes: GP/3T-1203, GP/3T-1204 (contraimpressão de GP/3T-1203) e GP/3T-I 205 a

Descriçâo: Cicatrizes foliares de aspecto arredondado e poligonal, de 2,0 $\mathrm{mm}$ de diâmetro, com feixe vascular, raramente visivel, de posição central. As estriações finas entre as cicatrizes quando presentes, são transversais a ligeiramente inclinadas em relação ao alongamento do caule. As cicatrizes estão dispostas numa helicoidal de ângulo pouco maior que o do Tipo A, em relação à horizontal. A distância entre uma cicatriz foliar e a próxima, numa helicoidal, é de cerca de 3,0 a $3,3 \mathrm{~mm}$. Na longitudional é de $5 \mathrm{~mm}$. Não se observam almofadas foliares, paricnos e lígula.
Comparação: Talvez sejam exemplares maiores e mais decortiçados, semelhantes ao Tipo A, sendo igualmente semelhantes às formas de $\mathrm{Ha}$ plostigma. Entretanto, apresentam ainda certa semelhança com Cyclostigma pacifica de JoNGMANS (1954:PL.23, fig. 29 cc).

\section{Cicatrizes Foliares Tipo C Est. II fig. 9 e 10,}

Espécime: GP/3T-1206

Descrição: Cicatrizes foliares de aspecto losangular alongado e apertado, de mais ou menos 6 $\mathrm{mm}$ de comprimento por $1,5 \mathrm{~mm}$ de largura, com feixe vascular de posição central, razoavelmente nitido. Sulcos profundos, anastomosantes entre as cicatrizes foliares, bastante próximas umas das outras. As cicatrizes estão dispostas numa helicoidal de ângulo muito pequeno em relação à horizontal, dando um aspecto pseudo-verticilado. A distância de um ponto central ao outro é de $2 \mathrm{~mm}$. Não há almofadas foliares, paricnos e lígula. $\mathrm{Na}$ longitudinal, a distância seria de $9 \mathrm{~mm}$.

Comparação: Assemelha-se pela forma de suas cicatrizes ao holótipo de Haplostigma em

\section{ESTAMPA 1}

Fig. 1 - Espécime GP/3T-1.201 - Cicatrizes foliares Tipo A.

Fig. 2 - Espécime GP/3T-1.202 - Cicatrizes foliares Tipo A.

Fig. 3 - Molde em látex do espécime GP/3T-1.203 - Cicatrizes foliares Tipo B.

Fig. 4 -Espécime GP/3T-1.203 - Cicatrizes foliares Tipo B.

Fig. 5 - Molde em látex do espécime GP/3T-1.204 - Cicatrizes foliares Tipo B.

Fig. 6 - Espécime GP/3T-1.204 - Cicatrizes foliares Tipo B contra impressão do GP/3T-1.203.

Fig. 7 - Molde em látex do espécime GP/3T-1.205a. Cicatrizes foliares Tipo B.

Fig. 8 - Espécime GP/3T-1.205a. Cicatrizes foliares Tipo B.

\section{ESTAMPA ॥}

Fig. 9 - Espécime GP/3T-1.206 - Cicatrizes foliares Tipo C.

Fig. 10 - Molde em látex do espécime GP/3T-1.206 - Cicatrizes foliares Tipo C.

Fig. 11 - Espécime GP/3T-1.213 - Folha destacada de Licófita.

Fig. 12 - Espécime GP/3T-1.207 - Cicatrizes foliares Tipo D.

Fig. 13 - Espécime GP/3T-1.210 - Cicatrizes foliares Tipo D.

Fig. 14 - Molde em látex do espécime GP/3T-1.212 - Cicatrizes foliares Tipo D.

Fig. 15 - Espécime GP/3T-1.212 - Cicatrizes foliares Tipo D.

Fig. 16 - Espécime GP/3T-1.205b - Cicatrizes foliares Tipo E. 


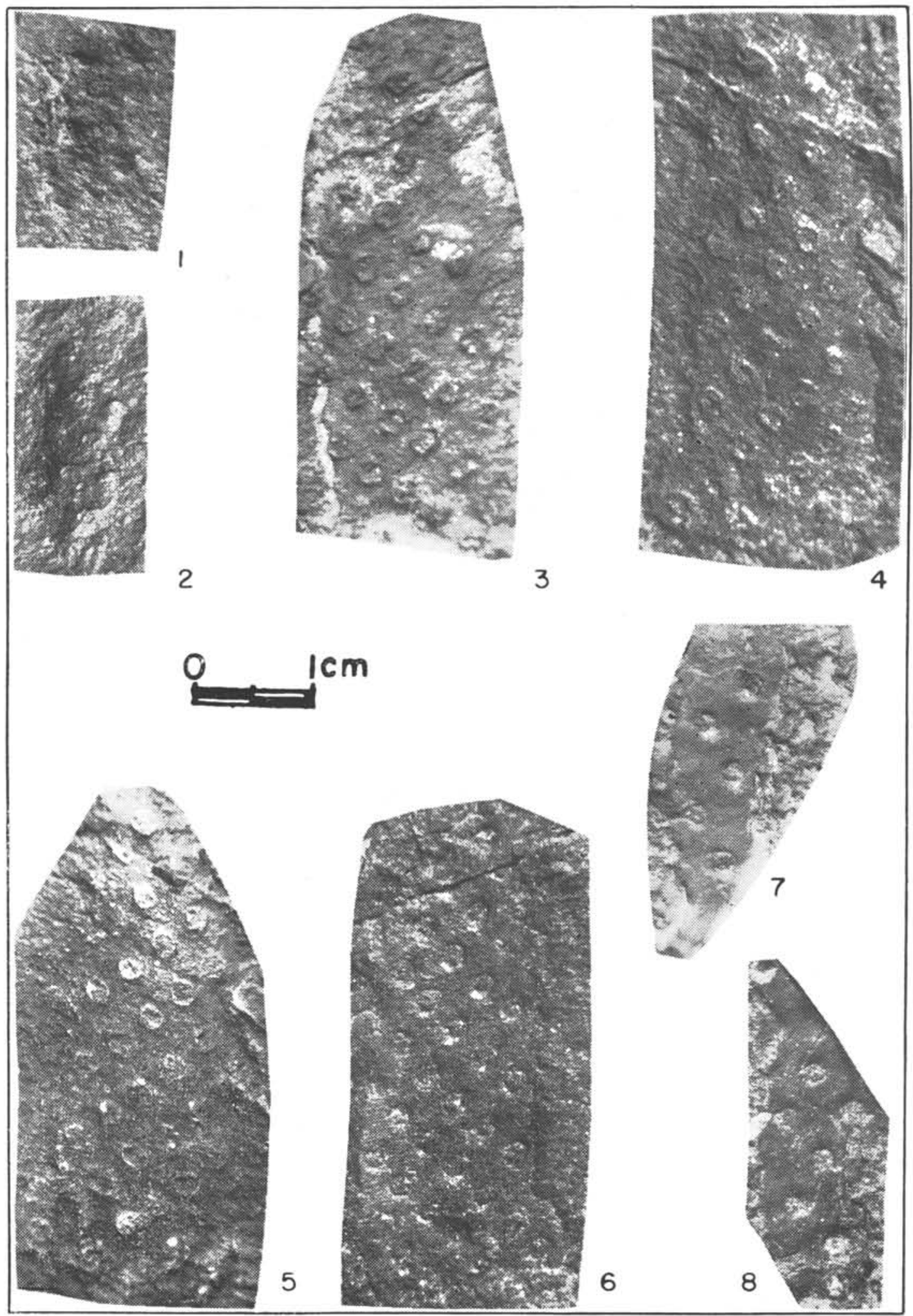




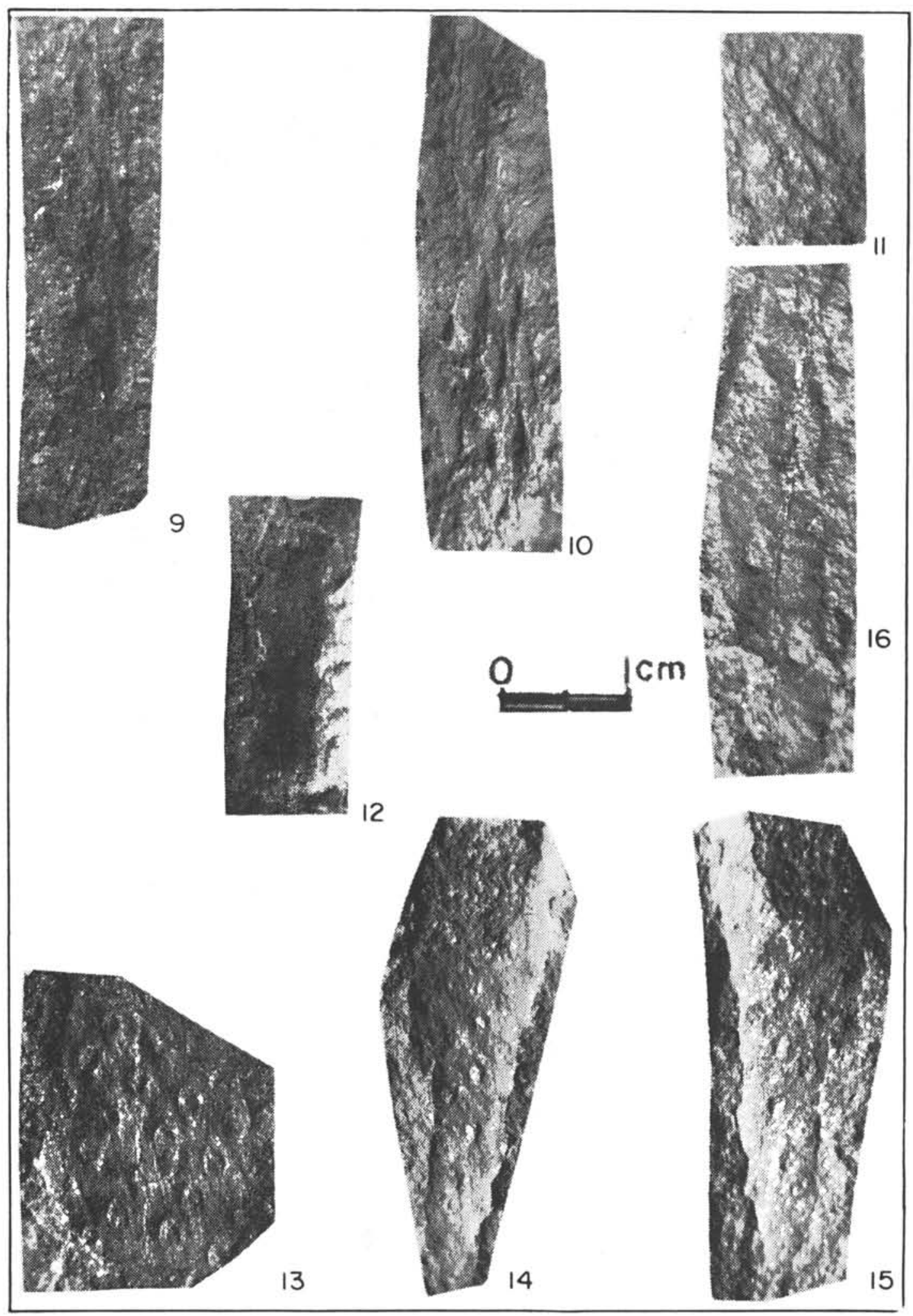


Plumstead (1967:PL XIII, fig. 1.). Também apresenta certa semelhança com Protolepidodendron.

\section{Cicatrizes Foliares do Tipo D Est. II fig. 12 a 15}

Espécimes: GP/3T-1207, GP/3T-1208, GP/3T. 1209, GP/3T-1210, GP/3T-1211a, GP/3T-1211 b e GP/3T-1212.

Descrição: Cicatrizes foliares do tipo arredondado passando a losangular de cauda longa de 2 . $2,4 \mathrm{~mm}$ de largura por $4-5,6 \mathrm{~mm}$ de comprimento, com posição central de feixe vascular nem sempre nítida. Estriações finas longitudinais entre as cicatrizes estão dispostas numa helicoidal nitida.

A distância entre as cicatrizes foliares é de ordem de $2 \cdot 2,4 \mathrm{~mm}$. Não há almofadas foliares, paricnos e ligula. Na longitudinal, a distância entre cicatrizes é de 3 a $6 \mathrm{~mm}$.

Comparaçāo: Assemelha-se a uma Cyclostigma pacifica do tipo apresentado por JONGMANS (1954:PL 20, fig. $14 b_{2}$ e $14 b_{3}$. Apresenta ainda certa semelhança com Lepidosigillaria de CHALONER in BOUREAU, 1967, p. 691) contudo, sem almofada foliar propriamente dita.

\section{Cicatrizes Foliares do Tipo E} Est. II, fig. 16.

Espécime: $\mathrm{GP} / 3 \mathrm{~T}-1205 \mathrm{~b}$

Descrição: Cicatrizes foliares hexagonais a arredondadas, de $2 \times 1 \mathrm{~mm}$, com feixe vascular central, por vezes nitido. Cristas longitudinais fortes entre as cicatrizes. A disposição das cicatrizes é pseudo-verticilada. Distanciam-se de mais ou menos $2,5 \mathrm{~mm}$ na transversal e de cerca de $7 \mathrm{~mm}$ na longitudinal.

Comparaçāo: Tem certa semelhança com Sigillaria do grupo Eusigillaria, todavia não apresenta paricnos.

\section{Folhas destacadas de Licófitas \\ Est. II, fig 11}

Espécime: GP/3T-1213

Descrição: Folhas aciculares medindo $3,4 \mathrm{~mm}$ de largura na base e $16,0 \mathrm{~mm}$ de comprimento. Não se observa bem a nervação. Parecem ter sido coriáceas e aderidas ao caule por toda a base.

\section{Idade}

O caráter fragmentário e, sobretudo, a forma de fossilização limitam muito as considerações concretas sobre aspectos sistemáticos e, consequentemente, cronológicos. Contudo, considerando serem sedimentos palinologicamente estéreis e até o momento carentes de outros recursos para sua datação, as informações fornecidas pelas licófitas aqui estudadas, mesmo que pouco precisas, apresentam especial interesse.

Dois recursos são aqui utilizados: tentativa de correlação com formas semelhantes e a interpretação tendo em vista um modelo filogenético.

Conforme discutimos acima, há certa semelhança das licófitas aqui descritas com as formas Haplostigma irregulares Seward observadas no sistema do Cabo por Plumstead (1967) que as considerou como de idade devoniana. Entretanto, até o momento não existem evidências seguras que confirmem esta idade. As relações estratigráficas seriam compatíveis com uma idade um pouco mais recente e a própria flora descrita por PLUMSTEAD (Op. cit) poderia ser do Carbonífero Inferior.

Algumas licófitas aqui descritas, por outro lado, apresentam grande semelhança com formas de Cyclostigma pacifica descritas por JONGMANS (1954) as quais ocorrem no Carbonífero Inferior do Peru.

Levando em conta um modelo filogenético, observa-se que a forma de cicatriz foliar já relativamente bem definida, a disposição mais helicoidal das folhas, a forma acicular das folhas (poucas foram observadas) podem implicar em um estágio evolutivo relativamente avançado 
considerando as licófitas mais primitivas do Devoniano. A própria constância na ausência de folhas, caso não decorra da fossilização, argumentaria na mesma linha.

Dessa forma, a idade aproximada dos niveis da formação Arayán, que contém essas li- cófitas, seria do Devoniano Superior a Carbonifero Inferior. Numa tentativa de maior precisão, poder-se-ia sugerir uma idade carbonifera inferior. Entretanto outras evidências deverão ser exploradas, para uma atribuição cronológica mais segura.

\title{
BIBLIOGRAFIA
}

AGUIRRE, L et alli - 1974 - Andean magmatism: its paleogeographic and structural setting in the central part (309-35.S) of the Southern Andes. - Pacific Geology, 8: 1-38

BOUREAU, E. - 1967 - Traité de Paléobotanique - 845 p. - Masson et Cie , Ed.

CECIONI, G. - 1974 - Flysch devonico y orogenesis bretonica em Chile. Univ. de Chile. Fac. de Cien. Fís. y Matemáticas Depto. de Geologia - Publicacion no 42:19-21.

CHALONER. W.G. - Lycophyta - in BOUREAU E. Traité de Paléobotanique - pp. 437-834 - Masson et Cie, Ed.

JONGMANS, W. - 1954 - The Carboniferous Flora of Peru. Bull. Brit. Mus. Nat. Hist. (Geol.) 2: 191-223, 1954.

PLUMSTEAD, E. - 1967 - A general review of the Devonian fossil plants found in the Cape System of South Africa. Paleontologia Africana, vol X: 1-83.

\section{PRIMEROS ESTUDIOS SOBRE PLANTAS FOSILES DE LA FORMACION LIBERTAD, PROVINCIA DE LA RIOJA, ARGENTINA ${ }^{2}$}

\author{
CARLOS L. AZCUY' \\ SYLVIA N. CESARI
}

Departamento de Ciências Geológicas, Universidad de Buenos Aires.

(1) Investigador del CONICET

(2) Contribución al PICG, Proy. n? 42 Upper Paleozoic of South America.

\begin{abstract}
The first results of a study of well-preserved fossil plants which were found in coal shales of the Libertad Formation, in the surroundings of Dique de los Sauces, are introduced in this paper. Fertile and sterile specimens of Asterotheca piatnitzkyi Frenguelli of the association which is almost exclusively composed by pecopterids and Cordaitales are described and illustrated. One species of Cordai-
\end{abstract}

